\title{
Discriminant analysis models for early detection of glaucomatous optic disc changes
}

\author{
Michele Iester, Jost B Jonas, Christian Y Mardin, Wido M Budde
}

\begin{abstract}
Aim-To evaluate and compare four different mathematical formulas for the early detection of morphometric optic nerve head changes in chronic open angle glaucoma.

Methods-The optic nerve heads of 161 patients with perimetrically defined glaucomatous optic nerve damage and of 194 normal subjects were examined by confocal laser scanning tomography. Using four formulas of linear discriminant analysis and the optic cup shape measure as the single optic disc variable, the predictive power of each of these methods was examined to differentiate between the normal eyes and the glaucoma eyes.

Results-The highest predictive power had an optic disc sector based formula, in particular in eyes with medium and large optic discs. This optic disc sector based formula was the one with the best agreement with the other formulas examined. It achieved a better predictability than any single optic disc variable evaluated.

Conclusions-Combining quantitative optic disc variables by discriminant analysis functions, the predictive power of semiautomatic quantitative optic nerve head evaluation can be improved by providing the ophthalmologist with a diagnostic score for the detection of glaucomatous optic nerve damage. Because of the pattern of glaucomatous neuroretinal rim loss, an optic disc sector based discriminant formula may have a higher diagnostic precision than other formulas in detecting early glaucomatous damage. (Br f Ophthalmol 2000;84:464-468)
\end{abstract}

Glaucomatous optic nerve damage leads to a multitude of morphological changes of the optic nerve head and to a great variety of functional deficits. The loss in function can be measured by achromatic or blue on yellow perimetry, flicker perimetry, colour vision testing, and many other psychophysical strategies. ${ }^{1}$ Most of the morphological alterations of the optic nerve head have been described semiquantitatively or qualitatively using the cup/ disc diameter ratio and qualitative variables such as the occurrence of disc haemorrhages and neuroretinal rim notches. ${ }^{2}$

Since the introduction of confocal laser scanning tomography, new technology has made possible the semiautomatic quantification of the topography optic nerve head..$^{3-8}$ Using this technique, a whole array of new quantitative variables has become available to detect early changes of the optic disc..$^{9-14}$ In an effort to increase the predictive value of the quantitative variables in differentiating normal eyes from eyes with early glaucomatous damage, mathematical equations combining various morphometric variables have been proposed to increase the ability to detect early morphological changes. ${ }^{9} 1012-15$

The purpose of this investigation was to evaluate and compare four of these mathematical models (which have already been calculated and tested in previous studies on different study populations in other glaucoma centres) on a new group of patients with glaucoma.

\section{Patients and methods}

The study included 161 patients with chronic open angle glaucoma with a mean age of 54.9 (SD 11.7) years (range 22-76 years) and a mean refractive error of -0.87 (2.72) dioptres (range -7.5-6.5 dioptres); and 194 normal subjects with a mean age of 44 (13.9) years (range 15-72 years) and a mean refractive error of -0.69 (1.93) dioptres (range $-7.0-4.0$ dioptres) with no significant difference between the two study groups in refractive error $(p=0.228)$. The differences in age were statistically significant $(p<0.001)$. Eyes with a myopic refractive error exceeding -8 dioptres were excluded owing to a differing optic disc morphology. ${ }^{10}$

The subjects in the normal control group were recruited from the administrative staff of the hospital who were having a routine ocular check up, or who came to the hospital for a prescription for glasses or for diagnosis and treatment of diseases not primarily of the optic nerve. All patients and subjects were examined as part of the Erlangen glaucoma registry. Only one randomly selected eye was taken from each subject and patient for statistical analysis.

Criteria for the diagnosis of open angle glaucoma were an open anterior chamber angle and glaucomatous visual field defects. A glaucomatous visual field defect was defined as a Octopus G1 field when mean visual field defect was more than $2 \mathrm{~dB}$ and corrected loss variance was greater than $4 \mathrm{~dB}^{2}$. All patients were examined perimetrically using the Octopus program G1 on the same day as the optic disc was evaluated. The rate of false positive or false negative results in the perimetric examination was less than $15 \%$ for all patients included in the study. The appearance of the optic nerve head was not taken as a diagnostic criterion. In the entire glaucoma group the mean defect was 7.52 (SD 5.1) dB (range 1.6$25.1 \mathrm{~dB}$ ) and corrected loss variance was 40.53 (37.61) $\mathrm{dB}^{2}$ (range 0.30-154.8). 
The glaucoma group included 76 patients with primary open angle glaucoma, 29 patients with secondary open angle glaucoma due to pseudoexfoliation syndrome, and 56 patients with normal pressure glaucoma. In primary open angle glaucoma, no obvious reason for elevation of the intraocular pressure could be detected. In the eyes with secondary open angle glaucoma, elevation of intraocular pressure was due to pseudoexfoliation of the lens ("pseudoexfoliative glaucoma"). It was characterised by a dandruff-like material on the lens zonules and the lens surface especially in its centre and its periphery separated by an intermediate clear zone, a secondary melanin dispersion with translucent defects in the parapupillary region of the iris pigment epithelium, often a hyperpigmentation of the anterior chamber angle, and a decreased facility to dilate the pupil. Criteria for the diagnosis of normal pressure glaucoma were maximal intraocular pressure readings equal to or less than $21 \mathrm{~mm} \mathrm{Hg}$ in at least two 24 hour pressure profiles obtained by slit lamp applanation tonometry and with measurements at 5 pm, 9 pm, midnight, 7 am, and noon. Ophthalmoscopy, medical history, and neuroradiological, neurological, and medical examinations did not reveal any reason, such as intrasellar or suprasellar tumours, retinal vessel occlusions, optic disc drusen, or non-arteritic anterior ischaemic optic neuropathy, for optic nerve damage other than glaucoma.

The optic nerve heads were morphometrically evaluated using the Heidelberg retina tomograph (HRT, Heidelberg Engineering, Heidelberg, Germany; software version 2.01). The Heidelberg retina tomograph is a confocal scanning diode laser with a wavelength of 670 nm. A series of 32 confocal images are obtained at consecutive focal planes, each 256 $\times 256$ pixels in size that the computer converts into a single topographic image. The depth of each topographic image series ranges between $0.5 \mathrm{~mm}$ and $4.0 \mathrm{~mm}$ in $0.5 \mathrm{~mm}$ increments depending on individual differences in the optic disc morphology. For each eye, three $10^{\circ}$ confocal scanning laser tomographic images were obtained; the mean was 28.0 (SD 17.6)

Table 1 Descriptive analysis

\begin{tabular}{|c|c|c|c|c|c|}
\hline & \multicolumn{2}{|c|}{$\begin{array}{l}\text { Normal } \\
(n=194)\end{array}$} & \multicolumn{2}{|c|}{$\begin{array}{l}\text { Glaucomatous } \\
(n=161)\end{array}$} & \multirow[b]{2}{*}{$p$ Value } \\
\hline & Mean & $S D$ & Mean & $S D$ & \\
\hline Age (years) & 44 & 13.9 & 54.9 & 11.7 & $<0.001$ \\
\hline Refractive error (dioptres) & -0.69 & 1.93 & -0.87 & 2.72 & 0.228 \\
\hline Mean defect $(\mathrm{dB})$ & 0.91 & 1.18 & 7.52 & 5.1 & $<0.001$ \\
\hline Corrected loss variance $\left(\mathrm{dB}^{2}\right)$ & 1.42 & 1.21 & 40.53 & 37.61 & $<0.001$ \\
\hline Global area $\left(\mathrm{mm}^{2}\right)$ & 2.79 & 0.81 & 2.63 & 0.62 & 0.021 \\
\hline Cup shape measure & -0.15 & 0.10 & -0.05 & 0.11 & $<0.001$ \\
\hline Rim volume $\left(\mathrm{mm}^{3}\right)$ & 0.43 & 0.19 & 0.18 & 0.13 & $<0.001$ \\
\hline High variation contour $(\mathrm{mm})$ & 0.40 & 0.10 & 0.37 & 0.25 & 0.036 \\
\hline Retinal nerve fibre thickness (mm) & 0.24 & 0.08 & 0.16 & 0.08 & $<0.001$ \\
\hline $\operatorname{Rim}$ area $\left(\mathrm{mm}^{2}\right)$ & 1.68 & 0.42 & 1.01 & 0.47 & $<0.001$ \\
\hline Inferior area below reference $\left(\mathrm{mm}^{2}\right)$ & 0.26 & 0.21 & 0.42 & 0.19 & $<0.001$ \\
\hline Inferior effective area $\left(\mathrm{mm}^{2}\right)$ & 0.49 & 0.22 & 0.53 & 0.16 & 0.03 \\
\hline Nasal mean high contour (mm) & 0.03 & 0.11 & 0.10 & 0.10 & $<0.001$ \\
\hline Temporal mean high contour (mm) & 0.22 & 0.11 & 0.18 & 0.12 & 0.002 \\
\hline Superior peak high contour (mm) & -0.07 & 0.09 & -0.02 & 0.12 & $<0.001$ \\
\hline Cup area $\left(\mathrm{mm}^{2}\right)$ & 1.12 & 0.86 & 1.60 & 0.74 & $<0.001$ \\
\hline Cup volume $\left(\mathrm{mm}^{3}\right)$ & 0.35 & 0.38 & 0.57 & 0.46 & $<0.001$ \\
\hline
\end{tabular}

$\mathrm{n}=$ number of eyes. $\mu \mathrm{m}$. The optic disc margin defined as the inner edge of Elshnig's ring was outlined by an experienced observer (CYM). In case of doubt, an optic disc photograph taken on the same day as the HRT image was simultaneously projected to better visualise the border of the optic disc. The technique, including its reproducibility and reliability, has already been described in detail elsewhere. ${ }^{3-14}$ The HRT variables were measured for the optic disc as a whole and in four separate disc sectors. The right angled superotemporal sector and the right angled inferotemporal sector were tilted 15 degrees temporal to the vertical optic disc axis. ${ }^{16}$ The temporal horizontal disc sector with 60 degrees and the nasal sector with 120 degrees covered the remaining area. This disc sectioning is different from previous studies in which the optic nerve head was divided into six sectors (superotemporal, superonasal, nasal, inferonasal, inferotemporal, and temporal). ${ }^{17}$

We used the variables disc area or global area, cup shape measure (CSM), rim volume or volume above reference (RV), height variation contour (HVC), retinal nerve fibre layer thickness (RNFLt), rim area (RA), inferior area below reference (IABR), inferior effective area (IEA), nasal mean height contour (NMHC), temporal mean height contour (TMHC), superior peak height contour (SPHC), cup area or area below reference (CA), and cup volume or volume below reference $(\mathrm{CV})$.

Based on the confocal laser scanning measurements of the optic nerve head, we evaluated five formulas for early detection of morphological abnormalities:

(1) Use of a single HRT variable: cup shape measure (CSM)..$^{5}$

(2) A discriminant analysis formula developed by Mikelberg and colleagues ${ }^{9}$ :

$\mathrm{A}=(\mathrm{RV} \times 1.951)+(\mathrm{HVC} \times 30.125)-$ $(\operatorname{corCSM} \times 28.521)-10.083$

$\mathrm{B}=(-9.039 \times \mathrm{RV})+(\mathrm{HVC} \times 37.370)-$ (corCSM 15.442) - 7.4211

[corrected CSM (cor CSM) = CSM + $(0.001981 \times(50-$ age $))]$

When $\mathrm{A}>\mathrm{B}$, the optic nerve head was considered to be normal, and when $\mathrm{A}<\mathrm{B}$, the optic disc was considered to be glaucomatous.

(3) A linear discriminant function developed by Bathija and colleagues ${ }^{12}$ :

$\mathrm{A}=[-3.722803-5.57 \times \mathrm{HVC}+11.78 \times$ RNFLt $-4.37 \times \mathrm{CSM}+1.85 \times \mathrm{RA}]$

(4) A sector based formula developed by Iester and colleagues ${ }^{18}$ and adjusted for varying sector size ${ }^{16}$ :

$\mathrm{A}=[10.068 \times \mathrm{IABR}-7.018 \times \mathrm{IEA}+4.181 \times$ $\mathrm{NMHC}+3.1 \times \mathrm{TMHC} \times 2.081 \times \mathrm{SPHC}$ $+6.094 \times \mathrm{CSM}-11.048 \times \mathrm{RV}+1828]$

(5) A linear discriminant function developed by Mardin and colleagues ${ }^{14}$ :

$\mathrm{A}=[-2.77+0.3 \times \mathrm{RA}+3.7 \times \mathrm{RV}+4.3 \times$ $\mathrm{RNFLt}-3.7 \times \mathrm{CSM}-3.1 \times \mathrm{CV}-0.9 \times$

CA]

The scores " $A$ " of these formulas were used to evaluate sensitivity and specificity. The total 
Table 2 Subgroup descriptive analysis

\begin{tabular}{|c|c|c|c|c|c|}
\hline \multirow[b]{2}{*}{ ONHs with a disc area $<2 \mathrm{~mm}^{2}$} & \multicolumn{2}{|c|}{$\begin{array}{l}\text { Normal } \\
(n=33)\end{array}$} & \multicolumn{2}{|c|}{$\begin{array}{l}\text { Glaucomatous } \\
(n=22)\end{array}$} & \multirow[b]{2}{*}{$p$ Value } \\
\hline & Mean & $S D$ & Mean & $S D$ & \\
\hline Age (years) & 41.8 & 13.6 & 52.2 & 12.1 & 0.003 \\
\hline Refractive error (dioptres) & -0.33 & 1.83 & -2.59 & 3.39 & 0.001 \\
\hline Mean defect $(\mathrm{dB})$ & 1.18 & 1.22 & 7.32 & 6.69 & $<0.001$ \\
\hline Corrected loss variance $\left(\mathrm{dB}^{2}\right)$ & 1.01 & 1.16 & 32.02 & 32.35 & $<0.001$ \\
\hline Global area $\left(\mathrm{mm}^{2}\right)$ & 1.79 & 0.16 & 1.73 & 0.23 & 0.099 \\
\hline Cup shape measure & -0.24 & 0.06 & -0.11 & 0.12 & $<0.001$ \\
\hline Rim volume $\left(\mathrm{mm}^{3}\right)$ & 0.45 & 0.14 & 0.20 & 0.13 & $<0.001$ \\
\hline High variation contour $(\mathrm{mm})$ & 0.40 & 0.10 & 0.40 & 0.13 & 0.456 \\
\hline Retinal nerve fibre thickness (mm) & 0.27 & 0.08 & 0.19 & 0.08 & 0.001 \\
\hline Rim area $\left(\mathrm{mm}^{2}\right)$ & 1.53 & 0.19 & 0.90 & 0.36 & $<0.001$ \\
\hline Inferior area below reference $\left(\mathrm{mm}^{2}\right)$ & 0.06 & 0.05 & 0.23 & 0.11 & $<0.001$ \\
\hline Inferior effective area $\left(\mathrm{mm}^{2}\right)$ & 0.24 & 0.08 & 0.33 & 0.10 & $<0.001$ \\
\hline Nasal mean high contour (mm) & -0.04 & 0.09 & 0.13 & 0.09 & $<0.001$ \\
\hline Temporal mean high contour (mm) & 0.20 & 0.12 & 0.27 & 0.12 & 0.019 \\
\hline Superior peak high contour (mm) & -0.12 & 0.09 & 0.00 & 0.11 & $<0.001$ \\
\hline Cup area $\left(\mathrm{mm}^{2}\right)$ & 0.26 & 0.21 & 0.82 & 0.40 & $<0.001$ \\
\hline \multirow[t]{2}{*}{ Cup volume $\left(\mathrm{mm}^{3}\right)$} & 0.05 & 0.06 & 0.23 & 0.22 & $<0.001$ \\
\hline & \multicolumn{2}{|c|}{$\begin{array}{l}\text { Normal } \\
(n=91)\end{array}$} & \multicolumn{2}{|c|}{$\begin{array}{l}\text { Glaucomatous } \\
(n=101)\end{array}$} & \\
\hline ONHs with a disc area between 2 and $3 \mathrm{~mm}^{2}$ & Mean & $S D$ & Mean & $S D$ & $p$ Value \\
\hline Age (years) & 44 & 13.3 & 55.3 & 11.7 & $<0.001$ \\
\hline Refractive error (dioptres) & -0.71 & 1.85 & -0.47 & 2.41 & $<0.001$ \\
\hline Mean defect $(\mathrm{dB})$ & 0.91 & 1.24 & 7.86 & 5.17 & $<0.001$ \\
\hline Corrected loss variance $\left(\mathrm{dB}^{2}\right)$ & 1.43 & 1.21 & 43.26 & 40.32 & $<0.001$ \\
\hline Global area $\left(\mathrm{mm}^{2}\right)$ & 2.50 & 0.28 & 2.50 & 0.26 & $<0.001$ \\
\hline Cup shape measure & -0.17 & 0.08 & -0.05 & 0.10 & $<0.001$ \\
\hline Rim volume $\left(\mathrm{mm}^{3}\right)$ & 0.45 & 0.19 & 0.18 & 0.14 & $<0.001$ \\
\hline High variation contour (mm) & 0.42 & 0.09 & 0.34 & 0.12 & $<0.001$ \\
\hline Retinal nerve fibre thickness (mm) & 0.26 & 0.07 & 0.16 & 0.07 & $<0.001$ \\
\hline Rim area $\left(\mathrm{mm}^{2}\right)$ & 1.66 & 0.32 & 1.00 & 0.43 & $<0.001$ \\
\hline Inferior area below reference $\left(\mathrm{mm}^{2}\right)$ & 0.20 & 0.11 & 0.39 & 0.15 & $<0.001$ \\
\hline Inferior effective area $\left(\mathrm{mm}^{2}\right)$ & 0.44 & 0.12 & 0.51 & 0.10 & $<0.001$ \\
\hline Nasal mean high contour (mm) & 0.03 & 0.11 & 0.10 & 0.09 & $<0.001$ \\
\hline Temporal mean high contour (mm) & 0.25 & 0.11 & 0.19 & 0.10 & $<0.001$ \\
\hline Superior peak high contour $(\mathrm{mm})$ & -0.06 & 0.11 & 0.00 & 0.10 & $<0.001$ \\
\hline Cup area $\left(\mathrm{mm}^{2}\right)$ & 0.87 & 0.43 & 1.46 & 0.53 & $<0.001$ \\
\hline \multirow[b]{3}{*}{ ONHs with a disc area $>3 \mathrm{~mm}^{2}$} & 0.25 & 0.18 & 0.50 & 0.32 & $<0.001$ \\
\hline & \multicolumn{2}{|c|}{$\begin{array}{l}\text { Normal } \\
(n=70)\end{array}$} & \multicolumn{2}{|c|}{$\begin{array}{l}\text { Glaucomatous } \\
(n=38)\end{array}$} & \\
\hline & Mean & $S D$ & Mean & $S D$ & $p$ Value \\
\hline Age (years) & 45.2 & 14.9 & 55.6 & 11.4 & $<0.001$ \\
\hline Refractive error (dioptres) & -0.83 & 2.07 & -0.95 & 2.75 & 0.394 \\
\hline Mean defect $(\mathrm{dB})$ & 0.80 & 1.09 & 6.69 & 3.9 & $<0.001$ \\
\hline Corrected loss variance $\left(\mathrm{dB}^{2}\right)$ & 1.59 & 1.20 & 37.11 & 31.69 & $<0.001$ \\
\hline Global area $\left(\mathrm{mm}^{2}\right)$ & 3.63 & 0.63 & 3.50 & 0.40 & 0.141 \\
\hline Cup shape measure & -0.09 & 0.08 & -0.01 & 0.10 & $<0.001$ \\
\hline Rim volume $\left(\mathrm{mm}^{3}\right)$ & 0.39 & 0.21 & 0.17 & 0.13 & $<0.001$ \\
\hline High variation contour (mm) & 0.39 & 0.11 & 0.43 & 0.46 & 0.239 \\
\hline Retinal nerve fibre thickness (mm) & 0.20 & 0.07 & 0.15 & 0.10 & 0.001 \\
\hline $\operatorname{Rim}$ area $\left(\mathrm{mm}^{2}\right)$ & 1.78 & 0.55 & 1.08 & 0.61 & $<0.001$ \\
\hline Inferior area below reference $\left(\mathrm{mm}^{2}\right)$ & 0.43 & 0.23 & 0.59 & 0.20 & $<0.001$ \\
\hline Inferior effective area $\left(\mathrm{mm}^{2}\right)$ & 0.68 & 0.20 & 0.71 & 0.12 & 0.191 \\
\hline Nasal mean high contour (mm) & 0.07 & 0.11 & 0.07 & 0.11 & 0.462 \\
\hline Temporal mean high contour (mm) & 0.19 & 0.10 & 0.10 & 0.13 & $<0.001$ \\
\hline Superior peak high contour $(\mathrm{mm})$ & -0.07 & 0.07 & -0.08 & 0.16 & 0.242 \\
\hline Cup area $\left(\mathrm{mm}^{2}\right)$ & 1.84 & 0.91 & 2.42 & 0.67 & $<0.001$ \\
\hline Cup volume $\left(\mathrm{mm}^{3}\right)$ & 0.62 & 0.47 & 0.95 & 0.62 & 0.001 \\
\hline
\end{tabular}

$\mathrm{n}=$ number of eyes; ONHs = optic nerve heads.

study group was subdivided by the size of the optic disc. In the subgroup with small optic discs, disc area was less than $2 \mathrm{~mm}^{2}$; in the subgroup with medium sized optic discs, the disc area ranged between $2 \mathrm{~mm}^{2}$ and $3 \mathrm{~mm}^{2}$; in the subgroup with large optic nerve heads, the disc area was larger than $3 \mathrm{~mm}^{2}$.

The data of the HRT variables used in the formulas and the visual field indices were analysed by a descriptive analysis. Student's $t$ test was used to compare the results between the two groups when the distribution of the data was normal. The Mann-Whitney nonparametric test was used instead, when the distribution of the data was non-parametric. A p
Table 3 Specificity, sensitivity, and diagnostic precision

\begin{tabular}{|c|c|c|c|c|c|}
\hline & $C S M$ & $M I K$ & $B A T H$ & SECT & $M A R D$ \\
\hline \multicolumn{6}{|c|}{ All the considered eyes: } \\
\hline Specificity & 50 & 65 & 74.7 & 91.8 & 46.4 \\
\hline Sensitivity & 80.8 & 84.5 & 85.1 & 70.2 & 94.4 \\
\hline $\begin{array}{l}\text { Diagnostic } \\
\text { precision }\end{array}$ & 63.9 & 73.8 & 79.4 & 82 & 68.2 \\
\hline \multicolumn{6}{|l|}{ DA $<2 \mathrm{~mm}^{2}$ : } \\
\hline Specificity & 90.9 & 93.9 & 97 & 100 & 81.8 \\
\hline Sensitivity & 54.6 & 86.4 & 90.9 & 54.6 & 86.4 \\
\hline $\begin{array}{l}\text { Diagnostic } \\
\text { precision }\end{array}$ & 76.4 & 90.9 & 94.6 & 81.8 & 83.6 \\
\hline \multicolumn{6}{|c|}{$2 \mathrm{~mm}^{2} \leqslant \mathrm{DA} \leqslant 3 \mathrm{~mm}^{2}:$} \\
\hline Specificity & 57.1 & 68.1 & 78 & 96.7 & 57.1 \\
\hline Sensitivity & 83.2 & 84.2 & 84.2 & 72.3 & 94.1 \\
\hline $\begin{array}{l}\text { Diagnostic } \\
\text { precision }\end{array}$ & 70.8 & 76.6 & 81.3 & 83.9 & 76.6 \\
\hline \multicolumn{6}{|l|}{$\mathrm{DA}>3 \mathrm{~mm}^{2}$ : } \\
\hline Specificity & 21.4 & 47.1 & 60 & 81.4 & 15.7 \\
\hline Sensitivity & 89.5 & 84.2 & 84.2 & 73.7 & 100 \\
\hline $\begin{array}{l}\text { Diagnostic } \\
\text { precision }\end{array}$ & 45.4 & 60.2 & 68.5 & 78.7 & 45.4 \\
\hline
\end{tabular}

$\mathrm{DA}=$ disc area; $\mathrm{HRT}=$ Heidelberg retina tomograph CSM $=$ cup shape measure; MIK = Mikelberg formula; BATH = Bathija formula; SECT = sector formula Iester formula); MARD = Mardin formula.

value less than 0.05 was considered to be statistically significant. Sensitivity, specificity, and diagnostic precision were calculated for all the methods examined. The kappa statistic was used to study the agreement among the five different methods and between the methods. The kappa measures change corrected agreement on a scale of -1.0 to 1.0 , with 1.0 indicating perfect agreement. We used weights suggested by Landis and $\mathrm{Koch}^{19}$ : kappas of 0.0 or less were considered poor; 0.0 to 0.2 , slight; 0.21 to 0.4 , fair; 0.41 to 0.6 , moderate; 0.61 to 0.8 , substantial; and 0.81 to 1.0 , almost perfect.

\section{Results}

The glaucoma group and the normal group, and the respective subgroups, differed significantly in all HRT variables measured (Tables 1 and 2). Sensitivity, specificity, and diagnostic precision of the four formulas ranged between $50 \%$ and $94 \%$ (Table 3). The sector based formula had the highest diagnostic precision and highest specificity compared with all other formulas. Highest sensitivity was achieved with Mardin's formula. The worst results were obtained by the single variable "cup shape measure". Using kappa statistics, a kappa of 0.57 was found among the five methods with a standard error of 0.01 and a $95 \%$ confidence interval between 0.54 and 0.59 . Among all five formulas examined, the sector formula had the highest agreement with all the other methods (Table 4).

Dividing the study groups into three subgroups according to the disc area, sensitivity, specificity, and diagnostic precision of the five methods were higher in the subgroup with small sized optic discs than in the two other subgroups (Table 3). In the small optic disc group subgroup, the Mikelberg formula and Bathija formula gave the best results. In the subgroup with medium sized optic discs and in the large optic disc size subgroup, the sector formula obtained the best results. 
Table 4 Kappa statistic

\begin{tabular}{llll}
\hline & $\begin{array}{l}\text { Kappa } \\
\text { value }\end{array}$ & $\begin{array}{l}\text { Standard } \\
\text { error }\end{array}$ & $\begin{array}{l}95 \% \text { CI } \\
\text { of kappa }\end{array}$ \\
\hline Cup shape measure $v$ Mikelberg formula & 0.53 & 0.03 & $0.46-0.59$ \\
Cup shape measure $v$ Bathija formula & 0.61 & 0.03 & $0.55-0.68$ \\
Cup shape measure $v$ sector based formula & 0.78 & 0.03 & $0.72-0.83$ \\
Cup shape measure $v$ Mardin formula & 0.46 & 0.04 & $0.39-0.53$ \\
Mikelberg formula $v$ Bathija formula & 0.62 & 0.03 & $0.56-0.68$ \\
Mikelberg formula $v$ sector based formula & 0.74 & 0.03 & $0.69-0.79$ \\
Mikelberg formula $v$ Mardin formula & 0.54 & 0.03 & $0.48-0.61$ \\
Bathija formula $v$ sector based formula & 0.78 & 0.03 & $0.73-0.83$ \\
Bathija formula $v$ Mardin formula & 0.65 & 0.03 & $0.59-0.71$ \\
Mardin formula $v$ sector based formula & 0.83 & 0.02 & $0.78-0.87$ \\
\hline
\end{tabular}

\section{Discussion}

Confocal laser scanning tomography is a technique for computerised analysis of the optic nerve head providing the ophthalmologist with new variables which have so far not been measurable. Recent studies evaluated sensitivity, specificity, and diagnostic precision of these new quantitative optic disc variables for the differentiation of normal eyes and eyes with glaucomatous optic nerve damage. Depending on the individual composition of the study groups in these investigations, the quantitative optic disc variables were ranked according to their diagnostic precision.

Using ROC curves, Iester and colleagues showed that the variable cup shape measure was the best HRT variable to differentiate their normal eyes from eyes with glaucomatous visual field defects. ${ }^{20}$ Correspondingly, Uchida and co-workers reported that the variable cup shape measure was the best variable to detect glaucomatous optic nerve damage. In Uchida's study, cup shape measure was even better than a combination of variables and similar to a trained neural network. ${ }^{10}$ Mikelberg and colleagues introduced all global variables measured by the confocal laser scanning system in a discriminant analysis function and obtained a discriminant formula. ${ }^{9}$ This formula was tested in a second study group which was different from the study group which was used to create the formula, and the results were similar. ${ }^{11}$ In a similar strategy, Bathija and co-workers used a linear discriminant analysis to distinguish subjects with normal visual field from glaucomatous patients with visual field defects. ${ }^{12}$ Additionally, Bathija and colleagues tested the formula calculated by Mikelberg et al and obtained a relatively high diagnostic precision. ${ }^{9}{ }^{12}$ The Mikelberg formula, however, did not reach the same diagnostic precision as the Bathija formula, probably because the Bathija formula, in contrast with the Mikelberg formula, was created and tested on the same study population.

Despite differences in the study populations and in the equation of the discriminant functions, these studies showed that the predictive power of confocal scanning laser tomographic examination of the optic nerve head could be increased by combining various optic disc variables. As a next step, we compared these formulas with each other in examining a new study population which was different from the study groups which were used to calculate the formulas.
We found that the sector based formula had the highest diagnostic precision of $82.0 \%$ (Table 3). This held true in particular for the subgroup with the medium to large optic discs and the subgroup with large optic nerve heads (Table 3). This may be because in the early stages of the disease, glaucomatous optic nerve damage leads to morphological changes predominantly in the inferior and superior disc regions, ${ }^{21}$ which will be detected more sensitively in a sector based strategy than in a strategy examining the whole optic disc area. It agrees with previous computerised $\mathrm{ONH}$ analysis and planimetric studies of optic disc photographs in which the neuroretinal rim area measured separately in the temporal inferior and temporal superior disc sectors achieved higher correlation coefficients than the whole neuroretinal rim area when correlated with the visual field damage. ${ }^{13}{ }^{22}$ Correspondingly, the sector based formula had a relatively low diagnostic precision in the subgroup of small optic discs (Table 3), in which often an optic cup is not present and the division of the neuroretinal rim into different disc sectors is artificial. ${ }^{22}$

The differences in the diagnostic precision between the four formulas tested in the present study were not very marked (Table 3 ). Taking into account the pattern of glaucomatous neuroretinal rim loss, ${ }^{21}$ however, with the most pronounced changes in the inferior and superior disc regions in the early stages, the sector based formula or a modification of it may be the most useful one for the early detection of glaucoma. This may hold true especially for glaucomatous eyes with focal damage of the optic nerve leading to neuroretinal notches which are typically located in the temporal inferior or temporal superior disc sectors. ${ }^{16}$ Another finding supporting the sector based formula was that it was the one having the best agreement with the other methods (Table 4).

Despite the increase in diagnostic precision obtained by calculating the discriminant formulas, the diagnostic precision of the confocal laser scanning tomographic measurements of the optic nerve head were still relatively low for clinical conditions. The main reason may be the pronounced interindividual variability for all optic disc variables measured in the normal population. Similar results have been obtained when optic disc variables were measured by planimetry of stereo optic disc photographs in previous studies. ${ }^{16}$ The marked interindividual variability is typical of many other quantitative biological variables, such as body height and weight, and this may be the reason why the normal group and the glaucoma group showed a pronounced overlap in the quantitative optic disc variables. It shows that, for the early detection of glaucomatous optic nerve head damage, qualitative variables such as the presence of localised retinal nerve fibre layer defects, disc haemorrhages, or neuroretinal notches have some advantage, since their specificity is almost $100 \%$ and because they can be assessed ophthalmoscopically. Another reason for the overlap between the study groups in the present investigation may be ethnic differences in the appearance of the optic 
nerve head. Previous studies have shown that the optic disc size is smallest in white people, followed by Mexicans and Asians, and is largest in Afro-Americans. ${ }^{2}$ Thus, a possible reason for the five methods having such different results could be related to the optic disc size in and between the glaucoma and normal groups, as several authors have already shown. ${ }^{11} 132324$ Consequently, one of the questions which may be addressed in future studies is whether discriminant formulas may be adapted to the ethnic background of the patients. It has to be emphasised that the figures of sensitivity and specificity of the formulas tested in the present investigation could not be transferred directly to other study groups with a different composition. This could also hold true if the ethnic composition of the study groups differs since the appearance of the optic nerve head partially depends on the ethnic background. At the moment all five methods have some limitations and their clinical application could only be an indicative result to be added to all the other tests.

Supported by Italian Ophthalmological Society, by G Gaslini Institute (MI), and by Deutsche Forschungsgemeinschaft (SFB 539) (JBJ, CYM, WMB).

Proprietary interest: None.

1 Ritch R, Shields RB, Krupin T. The glaucomas. St Louis: Mosby 1989 , vol I.

2 Jonas JB, Budde WM, Panda-Jonas S. Ophthalmoscopic evaluation of the optic nerve head. Surv Ophthalmol 1999; 43:293-320.

3 Kruse FE, Burk ROW, Völcker HE, et al. Reproducibility of topographic measurements of the optic nerve head with topographic measurements of the optic nerve head with
laser tomographic scanning. Ophthalmology 1989;96:13204 .

4 Dreher AW, Tso PC, Weinreb RN. Reproducibility of topographic measurements of the normal and glaucomatou nerve head with the laser tomographic scanner. $A m \mathcal{F} O p h$ thalmol 1991;111:221-9.

5 Weinreb RN, Lusky M, Bartsch DU, et al. Effect of repetitive imaging on topographic measurements of the optic nerve head. Arch Ophthalmol 1993;111:636-8.

6 Cioffi GA, Robin AL, Eastman RD, et al. Confocal laser scanning ophthalmoscope: reproducibility of optic nerve head topographic measurements with the confocal scanning laser ophthalmoscope. Ophthalmology 1993;100:5762 .

7 Mikelberg FS, Wijsman K, Schulzer M. Reproducibility of topographic parameters obtained with the Heidelberg retina tomograph. F Glaucoma 1993;2:101-3.

8 Chauhan BC, LeBlanc RP, McCormick TA, et al. Test-retest variability of topographic measurements with confocal scanning laser tomography in patients with glaucoma and control subjects. Am f Ophthalmol 1994;118:9-15.

9 Mikelberg FS, Parfitt CM, Swindale NV, et al. Ability of the Heidelberg retina tomograph to detect early glaucomatous field loss. F Glaucoma 1995,4:242-7.

10 Uchida H, Brigatti L, Caprioli J. Detection of structural damage from glaucoma with confocal laser image analysis. Invest Ophthalmol Vis Sci 1996,37:2393-401.

11 Iester M, Mikelberg FS, Drance SM. The effect of optic disc size diagnostic precision with the Heidelberg retina tomograph. Ophthalmolgy 1997;104:545-8.

12 Bathija R, Zangwill L, Berry CC, et al. Detection of early glaucomatous structural damage with confocal scanning glaucomatous structural damage with confoca
laser tomography. F Glaucoma 1998;7:121-7.

13 Wollstein G, Garway-Heath DF, Hitchings RA. Identification of early glaucoma cases with the scanning laser ophthalmoscope. Ophthalmology 1998;105:1557-63.

4 Mardin CY, Horn FK, Jonas JB, et al. Preperimetric glaucoma diagnosis by confocal scanning laser tomography of the optic disc. Br F Ophthalmol 1999;83:299-304.

15 Jonas JB, Dichtl A. Optic disc morphology in myopic primary open-angle glaucoma. Graefes Arch Clin Exp Ophthalmol 1997;235:627-33.

16 Jonas JB, Gusek GC, Naumann GOH. Optic disc morphometry in chronic primary open-angle glaucoma. I. Morphometric intrapapillary characteristics. Graefes Arch Clin Exp Ophthalmol 1988;226:522-30.

17 Iester M, Swindale NV, Mikelberg FS. Sector based analysis of optic nerve head shape parameters and visual field indices in healthy and glaucomatous eyes. F Glaucoma 1997;6: ces in heal $370-6$.

18 Iester M, Parfitt CM, Swindale NV, et al. Sector-based analysis of Heidelberg retina tomograph (HRT) parameters in normal and glaucomatous eyes. Invest Ophthalmol Vis Sci 1997;38:S835 (Suppl).

19 Landis JR, Koch GG. The measurement of observer agreement for categorical data. Biometrics 1977;33:159-74.

20 Iester M, Mikelberg FS, Swindale NV, et al. ROC analysis of Heidelberg retina tomograph optic disc shape measure in glaucoma. Can $\mathcal{F}$ Ophthalmol 1997;32:382-8.

21 Jonas JB, Fernández M, Stürmer J. Pattern of glaucomatous neuroretinal rim loss. Ophthalmology 1993;100:63-7.

22 Jonas JB, Gusek GC, Naumann GOH. Optic disc morphometry in chronic primary open-angle glaucoma. II. Correlation of the intrapapillary parameters to visual field indices. Graefes Arch Clin Exp Ophthatmol 1988:226:531-8.

23 Jonas JB, Gusek GC, Naumann GOH. Optic disc, cup and neuroretinal rim size, configuration, and correlations in normal eyes. Invest Ophthalmol Vis Sci 1988;29:1151-8; correction: Invest Ophthalmol Vis Sci 1991;32:1893.

24 Mardin CY, Horn FK. Influence of optic disc size on the sensitivity of the Heidelberg Retina Tomograph. Graefes Arch Clin Exp Ophthalmol 1998;236:641-5. 